\title{
Estimation of bone conduction skull transmission by hearing thresholds and ear- canal sound pressure
}

Sabine Reinfeldt, Stefan Stenfelt and Bo Håkansson

\section{Linköping University Post Print}

\section{Tweet}

N.B.: When citing this work, cite the original article.

Original Publication:

Sabine Reinfeldt, Stefan Stenfelt and Bo Håkansson, Estimation of bone conduction skull transmission by hearing thresholds and ear-canal sound pressure, 2013, Hearing Research, (299), 19-28.

http://dx.doi.org/10.1016/j.heares.2013.01.023

Copyright: Elsevier

http://www.elsevier.com/

Postprint available at: Linköping University Electronic Press

http://urn.kb.se/resolve?urn=urn:nbn:se:liu:diva-94708 


\title{
Estimation of bone conduction skull transmission by hearing thresholds and ear-canal sound pressure
}

\author{
Sabine Reinfeldt ${ }^{\mathrm{a}}$, Stefan Stenfelt ${ }^{\mathrm{b}}$, Bo Håkansson ${ }^{\mathrm{a}}$ \\ a. Department of Signals and Systems, Division of Signal Processing and Biomedical \\ Engineering, Chalmers \\ University of Technology, SE-412 96 Göteborg, Sweden \\ b. Department of Clinical and Experimental Medicine, Division of Technical Audiology, \\ Linköping University, SE-581 83 Linköping, Sweden
}

\section{Corresponding author:}

Sabine Reinfeldt

Department of Signals and Systems

Chalmers University of Technology

SE-412 96 Göteborg, Sweden

Phone: +46 (0)31 7728063

Fax: +46 (0)31 7721782

Email: sabine.reinfeldt@chalmers.se 


\section{Abstract}

Bone conduction sound transmission in the human skull and the occlusion effect were estimated from hearing thresholds and ear-canal sound pressure (ECSP) measured by a probe tube microphone when stimulation was at three positions on the skull (ipsilateral mastoid, contralateral mastoid, and forehead). The measurements were done with the ear-canal open as well as occluded by an ear-plug. Depending on the estimation method, transcranial transmission at frequencies below $1 \mathrm{kHz}$ was between -8 and $5 \mathrm{~dB}$, around $0 \mathrm{~dB}$ at $1 \mathrm{kHz}$ that decreased with frequency to between -17 and $-7 \mathrm{~dB}$ at $8 \mathrm{kHz}$. The forehead transmission was, except at frequencies between 1 and $2 \mathrm{kHz}$, similar to that proposed in the standard ISO:389-3 (1994) when the threshold measurements were conducted with open ear-canals. Compared with the same measurements using hearing thresholds, the ECSP gave similar transmission results at most frequencies, but differed at 0.5, 0.75, 2 and $3 \mathrm{kHz}$. One probable reason for the differences between thresholds and ECSP measures was a significant perception improvement (lower thresholds) when the stimulation was at the ipsilateral mastoid that was not found at the other positions. This improvement, which also was present in the occlusion effect data, was hypothesized to originate in greater sensitivity of the cochlea for vibration in line with the ipsilateral stimulation direction than from other directions. 

Abbreviations
$\mathrm{AC}$ - air conduction
$\mathrm{BC}$ - bone conduction
BEST - balanced electromagnetic separation transducer
ECSP - ear-canal sound pressure
RETFL - reference equivalent threshold force level
SD - standard deviation 


\section{Introduction}

Recently, there have been several studies investigating sound transmission in the human skull, also known as bone conduction (BC) sound transmission, by measurements of skull vibrations (Eeg-Olofsson et al., 2011; Håkansson et al., 2010; Stenfelt and Goode, 2005a). The rationale for these studies is that the vibration of the cochlea is related to a hearing perception, and the measurement of cochlear vibration enables a fast estimation of the $\mathrm{BC}$ excitation with greater level and frequency resolution compared with the same measurements done by hearing thresholds. Also, the problem with high variability associated with BC thresholds is avoided. The caveat is that the relation between hearing thresholds and cochlear vibration have to date not been directly determined. So far, the validity of the assumption is based on similar effects seen in vibration and threshold estimates, for example transcranial attenuation estimates (Stenfelt, 2012). Another caveat is that most studies of cochlear vibrations were done in cadavers (Eeg-Olofsson et al., 2008; Eeg-Olofsson et al., 2011; Stenfelt and Goode, 2005a) or dry skull specimens (Stenfelt et al., 2000) as the vibration of the cochlea is generally not easily accessible in live humans.

One possible problem with the idea of cochlear vibration being directly related to perception of $\mathrm{BC}$ sound is that $\mathrm{BC}$ perception is not a single phenomenon but caused by several mechanisms, all contributing to the final BC perception (Stenfelt and Goode, 2005b; Stenfelt, 2011; Tonndorf, 1966; von Békésy, 1960). Even if the ultimate excitation in the cochlea is a traveling wave on the basilar membrane (Stenfelt et al., 2003b; Stenfelt, 2007; von Békésy, 1932), there are contributors that may not be directly related to the vibration of the cochlea. Such contributors can be the sound in the ear-canal during BC stimulation (Bárány, 1938; Stenfelt et al., 2003a) or the inertial effects of the middle ear ossicles (Homma et al., 2009; Huizing, 1960; Stenfelt et al., 2002); although possible, it is not clear that they are related to the cochlear vibration. The middle 
ear ossicles may contribute to the BC perception at mid-frequencies (1.5-3 kHz) (Stenfelt, 2006), while the outer ear only is believed to be a main contributor at low frequencies (below $2 \mathrm{kHz}$ ) when the ear-canal is occluded (Stenfelt et al., 2003a; Stenfelt et al., 2007). Another contributor that seems unrelated to the cochlear vibration is the proposed dynamic pressure transmission from the skull interior (e.g. the cerebrospinal fluid) to the cochlea (Freeman et al., 2000). However, it has not been shown to be a significant contributor to $\mathrm{BC}$ hearing in a healthy ear (Stenfelt, 2011).

To date, there are no studies that have directly related the cochlear vibration to a BC perception. In an attempt to distinguish the importance of different contributors to BC sound, Ito et al. (2011) measured the hearing thresholds and teeth vibration during BC stimulation at different positions of the skull (including the eye). They concluded, from comparisons of hearing thresholds and teeth vibrations, that the $\mathrm{BC}$ perception is not directly related to vibration of the skull bones. However, it is questionable how well the teeth mimic the vibration of the cochlea during BC stimulation. A more often used method is the indirect comparison of skull vibration data and hearing threshold data in cross head transmission estimates. In these studies masking may be a problem in the perceptual estimate of transcranial transmission and sensitivity from the forehead. Others have used subjects with unilateral deafness to circumvent the uncertainty associated with masking procedures. Recently, the transcranial attenuation was estimated for two stimulation positions (one close to the ear-canal opening and the other further back) by hearing thresholds (Stenfelt, 2012). In the same study the results were compared with estimations from vibration of the cochlea in cadaver heads using one-dimensional velocity measurements (Eeg-Olofsson et al., 2011) and three-dimensional acceleration measurements (Stenfelt and Goode, 2005a). According to Stenfelt (2012), average perceptual measures were close to the vibration estimates at 
frequencies between 0.8 and $6 \mathrm{kHz}$. In Stenfelt and Goode (2005a) the vibration data of the cochlear promontory was compared to the proposed sensitivity difference between ipsilateral mastoid and forehead BC stimulation according to the standard ISO:389-3 (1994). The worse sensitivity at the forehead of 8 to $14 \mathrm{~dB}$ corresponded well with the cochlear promontory vibration data: at the lower frequencies (below $3 \mathrm{kHz}$ ) one-dimensional cochlear vibration predicted the response, while at higher frequencies three-dimensional vibration data gave a better estimate.

Most BC data are obtained with the stimulation at the mastoid or at the forehead as these positions are used clinically and perceptual threshold levels are standardized (ISO:389-3, 1994). Although not thoroughly investigated, it is plausible that different stimulation positions on the skull result in different vibration pattern of the cochlea and surrounding structures that alter the relative contributions of the mechanisms resulting in a perception of BC sound. An indication of this phenomenon is that, for several different middle ear lesions, approximately $5 \mathrm{~dB}$ worse BC thresholds at frequencies between 0.5 and $4 \mathrm{kHz}$ were obtained with stimulation at the mastoid compared with forehead (Dirks et al., 1969; Goodhill et al., 1970; Studebaker, 1962). Hence, the middle ear component of BC sound may be more important when stimulation is in line with the vibration of the ossicles (mastoid) than when it is perpendicular (forehead). Another more obvious difference due to stimulation position is when the vibration is applied at the soft tissues rather than at the skull bone (Ito et al., 2011). Such stimulation may excite the suggested pathways for BC perception significantly differently and the vibration of the bony parts surrounding the cochlea may be unrelated to the BC perception.

Even if the vibration of the cochlear promontory is related to BC perception, its vibrational response is difficult to obtain in a normal human ear. An entity that is relatively easy to measure 
is the ear-canal sound pressure (ECSP). It reflects the sound pressure in the ear-canal produced from vibrations of the surrounding structure. Since the ear-canal is close to the cochlea, one can hypothesize that the vibration pattern of the bony ear-canal is similar to the vibration of the cochlea, and, as a result, the ECSP may be used to estimate the cochlear perception during BC stimulation. Consequently, an alteration of the ear-canal bone vibration level equals the same ECSP level alteration. Moreover, the alteration of ECSP level is, according to the hypothesis, correlated to the cochlear vibration level change and corresponding BC perceptual level change. This concept is easily understood if the stimulation position is constant and only the stimulation level is altered: the same level alteration is seen in the ECSP level and perception level of the BC sound. However, it is not equally clear that it holds for an alteration of stimulation position. For example, when the BC stimulation position is moved from the forehead to the mastoid, the ipsilateral cochlear perception level is expected to increase as well as the ECSP level. But are the two increases equal? If so, the ear-canal may serve as an easily accessible way to estimate BC response by the way of ECSP measurements during BC stimulation. So even if the ECSP is not the primary cause for perception of BC sound in a normal ear (Bárány, 1938; Stenfelt et al., 2003a), the relative ECSP might reflect changes in BC perception.

The aim of the study is to investigate the relation between changes in ECSP and perception during BC stimulation when the stimulation position is altered. In addition, the change of the two entities is studied for both open and occluded ear-canals. A secondary aim is to investigate the importance of the contributors to BC perception as a function of stimulation position. This is accomplished by comparing relative results with the ear-canal open and occluded, and the occlusion effect with stimulation at different positions. 


\section{Material and methods}

\subsection{Subjects}

Twenty normal hearing subjects (ten male and ten female) with a median age of 28 years (23 to 43 years) participated voluntarily in the study. They all had otologically normal ears (ISO:226, 2003) with hearing thresholds equal or better than $20 \mathrm{~dB}$ HL and their inter-aural difference was maximum $15 \mathrm{~dB}$ in the frequency range 0.125 to $8 \mathrm{kHz}$.

\subsection{Measurement setup}

All measurements were conducted in a sound insulated room of $16 \mathrm{~m}^{3}$. The baseline air conduction (AC) hearing thresholds were first measured using an audiometer (Interacoustics AC40) with TDH39 headphones to verify the subject's hearing status. The BC hearing thresholds were obtained with the same audiometer but with the output routed through a power amplifier (Sony TA-N220) to a BC transducer, termed the BEST (Balanced Electromagnetic Separation Transducer) (Håkansson, 2003). This transducer is specially designed to, compared with the Radioear B71, provide higher stimulation levels with a minimum of nonlinear distortion and it is slightly smaller to reduce the airborne radiation. It comprises a circular interface of $175 \mathrm{~mm}^{2}$ enabling comparison with the ordinary B71 transducer.

The BC hearing thresholds were obtained with a pulsed Békésy procedure (ISO:8253-1, 2010) at the fixed frequencies $0.125,0.25,0.5,0.75,1.0,1.5,2.0,3.0,4.0,6.0$, and $8.0 \mathrm{kHz}$. The transducer was placed with a headband (steel spring arrangement) at one of three positions: (1) ipsilateral mastoid, (2) contralateral mastoid, and (3) the center of the forehead.

To isolate the test ear during BC stimulation, the non-test ear was masked with prerecorded pink noise provided by a CD player (Technics SL-PS740A) via a power amplifier (Rotel RB- 
976MkII) to an insert earphone (Etymotic Research ER-2). To minimize occlusion of the masked ear, the foam part of the ER-2 was reduced to three small "foam wings". This manipulated ER-2 foam plug was verified by measurements to not cause any occlusion effect. The masking level was set at a minimum $30 \mathrm{~dB}$ above the threshold level of the contralateral ear's AC threshold and was the same at all three BC stimulation positions.

The setup for measuring ECSP close to the TM during BC stimulation is shown in Fig. 1. The ECSP was measured with a 4-channel input, 2-channel output Brüel \& Kjær Pulse ver. 10.0 system. The same amplifier and BC transducer as with threshold estimation was used and the output from the signal analyzer was fed to the power amplifier and subsequently the BC transducer, see Fig. 1. Inputs to the signal analyzer were the output from the amplifier and the signal from the probe tube microphone (Etymotic Research ER-7C) representing the ECSP. A frequency response function of the probe tube microphone signal divided by the input to the BC transducer (output of the amplifier) was produced by the signal analyzer using a swept sine method with a frequency resolution of 24 frequencies per octave. The person equipped with the $\mathrm{BC}$ transducer and the probe tube microphone was located in the sound insulated room; the rest of the equipment was situated outside. 
Figure 1

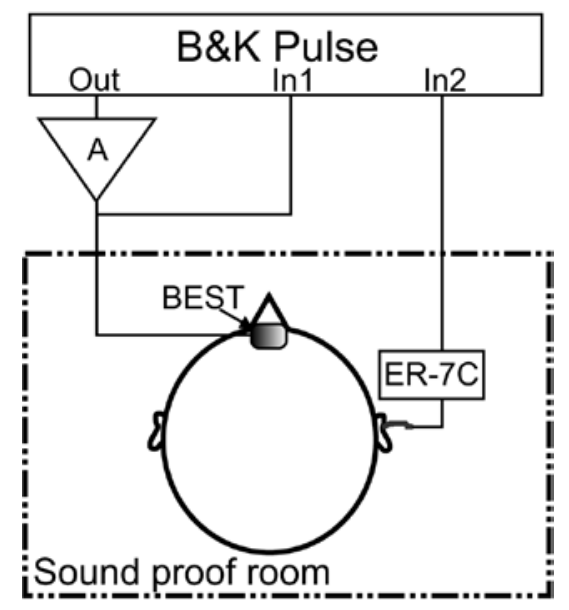

\subsection{Procedure of measuring the $B C$ hearing thresholds}

For all measurements, the test subject was sitting comfortable in a chair in the sound insulated room. During the threshold testing, the test subject was given a response button with the instructions to keep it pressed when a pulsed tone was heard, otherwise released. Using the pulsed Békésy test procedure, the BC hearing thresholds in the frequency range 0.125 to $8 \mathrm{kHz}$ (specific frequencies given above) were obtained with two ear-canal conditions ([1] open and [2] occluded (a foam ear-plug was inserted with its inner end $18 \mathrm{~mm}$ into the ear-canal relative to the tip of the tragus)) and three stimulation positions ([1] ipsilateral mastoid, [2] contralateral mastoid, and [3] center of forehead). Consequently, hearing thresholds were obtained for a total of six conditions. To minimize order effects, the tests were counterbalanced among the subjects. The measurement sequence was constructed to minimize the reposition of the occluding ear-plug. To assure BC hearing response from the test ear only, the opposite ear was masked with pink noise at a minimum $30 \mathrm{~dB}$ above the individual's hearing thresholds of the non-test ear using the modified insert earphone (ER-2) described above. 
The BC transducer was attached with a headband at the three stimulation positions providing a static force of 4 to 5 Newtons. Each position on the skull was marked before attaching the transducer resulting in well-defined stimulation positions. This allowed reposition of the transducer at the three stimulation positions as well as comparisons of the results between testsubjects. Ipsilateral stimulation means that the BC transducer was attached at the mastoid portion of the temporal bone behind the test ear. During the positioning, it was made sure that the transducer did not touch the outer ear pinna. During contralateral stimulation, the BC transducer was applied to the mastoid portion of the opposite temporal bone and during forehead stimulation the BC transducer was applied at the center of the forehead.

\subsection{Procedure of measuring the ear-canal sound pressure}

The ECSP during BC stimulation was measured with the probe tube microphone in the frequency range 0.1 to $10 \mathrm{kHz}$ for the same conditions as the thresholds described above. To keep the conditions the same for the ECSP measurements and the threshold measurements, the ECSP measurement was done directly before or after the threshold test of a specific condition.

For open ear-canals, the microphone probe tube was inserted $25 \mathrm{~mm}$ relative to the tip of the tragus (in front of the ear-canal). After the probe tube was inserted a syringe with air was used to blow air through the probe tube to clean it from fluid or wax that might occlude the opening. This procedure ensured an open tube after the insertion. For occluded ear-canals, a foam ear-plug containing two open canals was inserted with its inner end $18 \mathrm{~mm}$ into the ear-canal relative to the tip of the tragus. The probe tube of the probe microphone was inserted through one of the earplug canals and air was blown through the probe tube with a syringe ensuring an open tube; the other canal of the ear-plug was plugged. The insertion depth of the microphone probe tube 
through the ear-plug was approximately the same as for the open ear-canal enabling comparison of the two conditions. The stimulation positions were the same as for the threshold estimations.

\subsection{Transmission calculations}

The transcranial transmission was calculated as the BC hearing threshold with the stimulation at the ipsilateral mastoid minus the BC hearing threshold with the stimulation at the contralateral mastoid. Similarly, the estimated transcranial transmission with ECSP was obtained as the ECSP with contralateral stimulation relative to ipsilateral stimulation (Fig. 2a). The results of the ECSP measurements with a resolution of 24 frequencies per octave were integrated to 3 frequencies per octave (third-octave bands) with values in the frequency range of 0.125 to $8 \mathrm{kHz}$. The threshold data were measured at 11 frequencies: all in-between frequencies according to the above thirdoctave frequencies were interpolated from the original audiometric test frequencies. In a similar way, the transmission from the forehead was the BC hearing threshold for ipsilateral relative to forehead stimulation and the ECSP for forehead relative to ipsilateral stimulation (Fig. 2b).

Figure 2
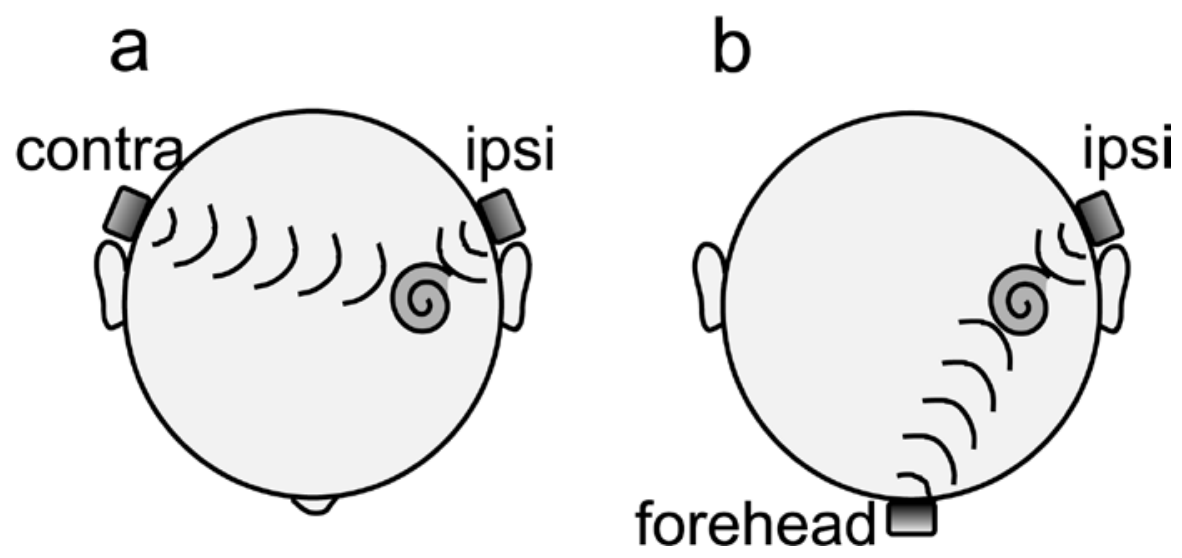


\subsection{Occlusion effect calculations}

Since the transcranial transmission and the transmission from the forehead were measured both with open and occluded ear-canals, the occlusion effect was calculated and compared for the three stimulation positions (ipsilateral and contralateral mastoids and the center of the forehead). The occlusion effect was for all stimulation positions obtained with the ear-plug $18 \mathrm{~mm}$ into the ear-canal in relation to the tragus, i.e. approximately $10 \mathrm{~mm}$ relative to the ear-canal opening. It should be noted that other depths and occlusion devices produce a different occlusion effect (Stenfelt and Reinfeldt, 2007). For the ECSP measurements, the occlusion effect was calculated as the occluded ECSP relative to the open ECSP. Since there are better (lower) BC hearing thresholds for occluded ear-canals, the perceived occlusion effect was calculated as the BC hearing thresholds for the open relative to the occluded ear-canal.

\subsection{Calibrations}

The probe microphone (ER-7C) was calibrated using a Brüel \& Kjær type 4134 1⁄2-inch microphone. According to the manufacturer, the 4134 microphone has a maximal level deviation of $2 \mathrm{~dB}$ in the frequency range 0.1 to $10 \mathrm{kHz}$. First, the sensitivity of the microphone was determined with a Brüel \& Kjær type 4230 sound level calibrator. Then the probe tube opening was placed $1 \mathrm{~mm}$ from the $1 / 2$-inch microphone, a sound field was introduced, and the calibration curve of the ER-7C was obtained for the frequency range 0.1 to $10 \mathrm{kHz}$.

The AC output for baseline hearing thresholds was calibrated according to ISO:389-1 (1998), while the output from the BC transducer was calibrated according to ISO:389-3 (1994). During the calibration, the BC transducer was placed on the Brüel \& Kjær type 4930 artificial mastoid 
where the output from the artificial mastoid was related to the dynamic output force of the BC transducer.

\subsection{Statistics}

Relative BC transmission in the skull (transcranial transmission and forehead transmission) was compared within subjects for hearing thresholds and ECSP for the two conditions open and occluded ear-canals using Student's paired t-test. Also, the estimates of the occlusion effect were compared for stimulation position (ipsilateral mastoid, contralateral mastoid, and forehead) and outcome measures (hearing thresholds and ECSP) using Student's paired t-test. Two levels of statistical significance are reported, $\mathrm{p}<0.05$ and $\mathrm{p}<0.01$; no adjustments for multiple comparisons were made. Only data at the 11 hearing threshold frequencies were included in the statistical analysis, no interpolated data were used. 


\section{Results}

\subsection{Transcranial transmission}

The transcranial transmission (stimulation at the contralateral mastoid compared with stimulation at the ipsilateral mastoid) results are shown in Fig. 3. Data are displayed as means from all 20 participants and the standard deviation (SD) is given for each estimate (means and SDs have the same line properties). The solid line is the result from the ECSP measurements with open earcanals while the dashed line originates from the ECSP measurements with occluded ear-canals. The other two curves are from the $\mathrm{BC}$ hearing thresholds; the dotted line shows the result with the ear-canal open and the dash-dotted line shows the result with the ear-canal occluded. The SDs of the estimates were fairly equal and no significant frequency dependence could be observed; all stayed primarily between 4 and $10 \mathrm{~dB}$ in the frequency range 0.1 to $10 \mathrm{kHz}$. At frequencies above $0.8 \mathrm{kHz}$, the means from the different methods were similar. Even if there were differences between the curves, some general findings could be observed in Fig. 3. At frequencies up to 1.5 $\mathrm{kHz}$ the transcranial transmission is close to $0 \mathrm{~dB}$ (falls between -8 and $5 \mathrm{~dB}$ ). Above this frequency and up to $8 \mathrm{kHz}$ there was a reduction in the transmission to between -7 and $-17 \mathrm{~dB}$ at the highest frequency. 
Figure 3
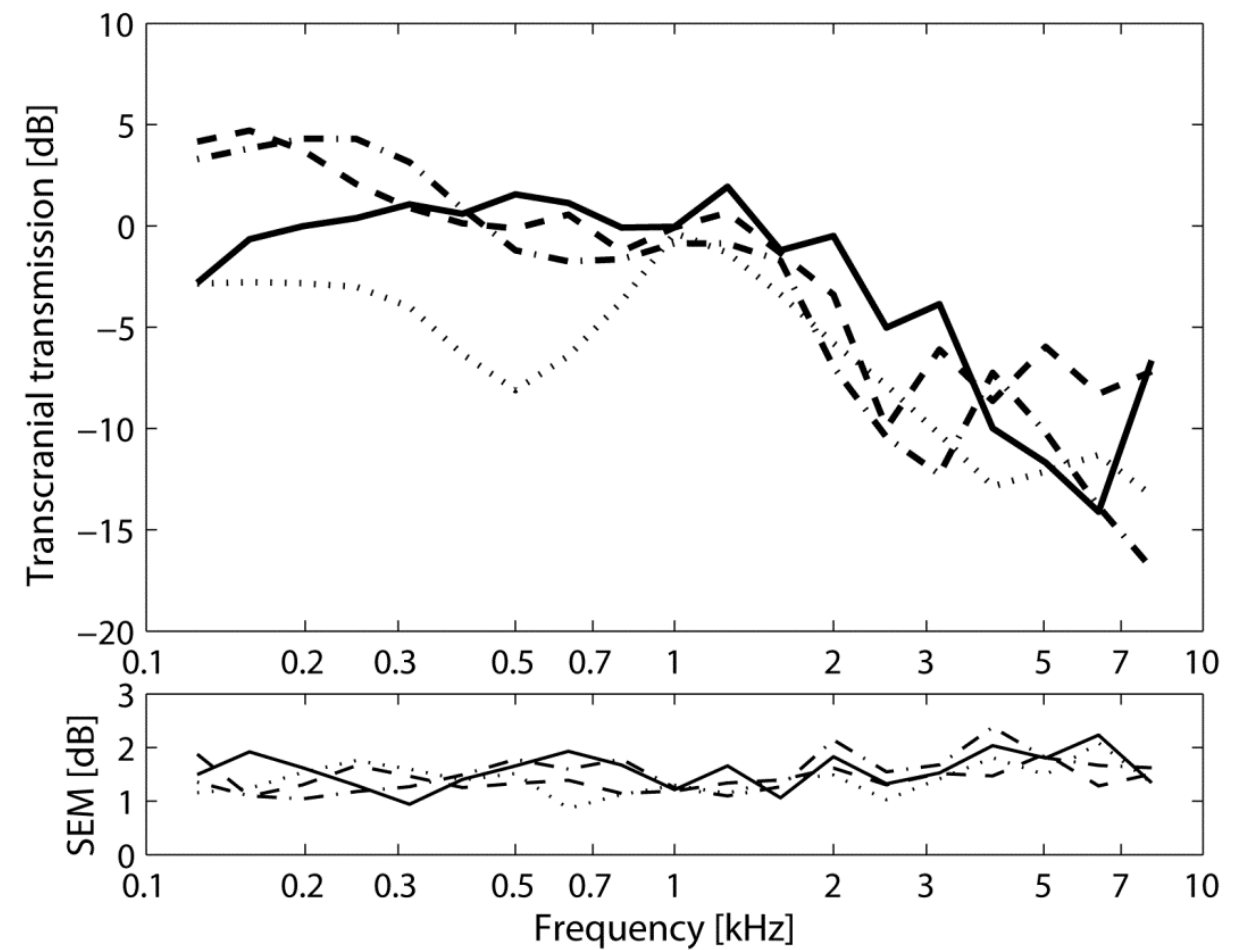

At low frequencies, there were some differences between the methods, especially between open and occluded ear-canals. The statistical significance of these differences, as determined by the ttest, is shown in Table 1 at the frequencies where the hearing thresholds were obtained. At and below $0.5 \mathrm{kHz}$, there was a statistically significant difference between open and occluded earcanals when the transcranial transmission was estimated by hearing thresholds (dotted and dashed-dotted lines in Fig. 3; $\mathrm{p}<0.01$ for all frequencies at and below $0.5 \mathrm{kHz}$ ). When the same comparison was done for the ECSP estimations of the transcranial transmission, only a statistical significant difference at the lowest frequency $(0.125 \mathrm{kHz})$ was found (solid and dashed lines in Fig. 3). At higher frequencies, above $0.5 \mathrm{kHz}$, only weak statistical significant differences $(\mathrm{p}<0.05)$ were seen for a few frequencies between the open and occluded ECSP estimates, and no statistical significant differences were seen for hearing thresholds (Table 1). 
Table 1: Statistical significance according to two-tailed paired t-test for BC thresholds and ECSP estimates of skull transmission. $T T$ = transcranial transmission; FT = forehead transmission; NS = non-significant.

\begin{tabular}{|c|c|c|c|c|c|c|c|c|}
\hline & \multicolumn{4}{|c|}{ Open vs Occluded } & \multicolumn{4}{c|}{ Hearing thresholds vs ECSP } \\
\hline & Hearing thresholds & \multicolumn{2}{|c|}{ ECSP } & \multicolumn{2}{c|}{ Open ear-canal } & \multicolumn{2}{c|}{ Occluded ear-canal } \\
\hline Frequency [Hz] & TT & FT & TT & FT & TT & FT & TT & FT \\
\hline 125 & $\mathrm{p}<0.01$ & $\mathrm{p}<0.01$ & $\mathrm{p}<0.01$ & $\mathrm{p}<0.01$ & NS & NS & NS & NS \\
\hline 250 & $\mathrm{p}<0.01$ & $\mathrm{p}<0.01$ & NS & NS & NS & NS & NS & NS \\
\hline 500 & $\mathrm{p}<0.01$ & $\mathrm{p}<0.01$ & NS & $\mathrm{p}<0.01$ & $\mathrm{p}<0.01$ & $\mathrm{p}<0.01$ & NS & NS \\
\hline 750 & NS & NS & NS & NS & $\mathrm{p}<0.05$ & $\mathrm{p}<0.05$ & NS & NS \\
\hline 1000 & NS & NS & NS & $\mathrm{p}<0.05$ & NS & NS & NS & NS \\
\hline 1500 & NS & NS & NS & NS & NS & NS & NS & NS \\
\hline 2000 & NS & NS & $\mathrm{p}<0.05$ & NS & $\mathrm{p}<0.01$ & $\mathrm{p}<0.05$ & NS & NS \\
\hline 3000 & NS & NS & $\mathrm{p}<0.05$ & NS & $\mathrm{p}<0.01$ & $\mathrm{p}<0.01$ & $\mathrm{p}<0.05$ & $\mathrm{p}<0.05$ \\
\hline 4000 & NS & $\mathrm{p}<0.01$ & NS & $\mathrm{p}<0.05$ & NS & NS & NS & NS \\
\hline 6000 & NS & NS & $\mathrm{p}<0.05$ & $\mathrm{p}<0.01$ & NS & NS & $\mathrm{p}<0.05$ & NS \\
\hline 8000 & NS & NS & NS & NS & $\mathrm{p}<0.01$ & NS & $\mathrm{p}<0.01$ & $\mathrm{p}<0.05$ \\
\hline
\end{tabular}

When comparing the results obtained by hearing thresholds and ECSP, fewer statistical significant differences were seen. With the ear-canal occluded the difference was statistically significant only at three of the highest frequencies $(3,6$ and $8 \mathrm{kHz})$ while there were more statistically significant differences when the ear-canal was open $(0.5-0.75 \mathrm{kHz}, 2-3 \mathrm{kHz}$, and 8 $\mathrm{kHz})$.

\subsection{Transmission from the forehead}

The mean results for the relative forehead transmission (stimulation at the forehead related to stimulation at the ipsilateral mastoid) are shown in Fig. 4. Exactly as for the transcranial transmission results, the solid and dashed lines are from ECSP measurements, open and occluded ear-canals, respectively. The BC hearing threshold results for open and occluded ear-canals are 
the dotted and the dash-dotted lines, respectively. Also included in Fig. 4 is the SD where the line properties are the same as for the mean results.

Figure 4

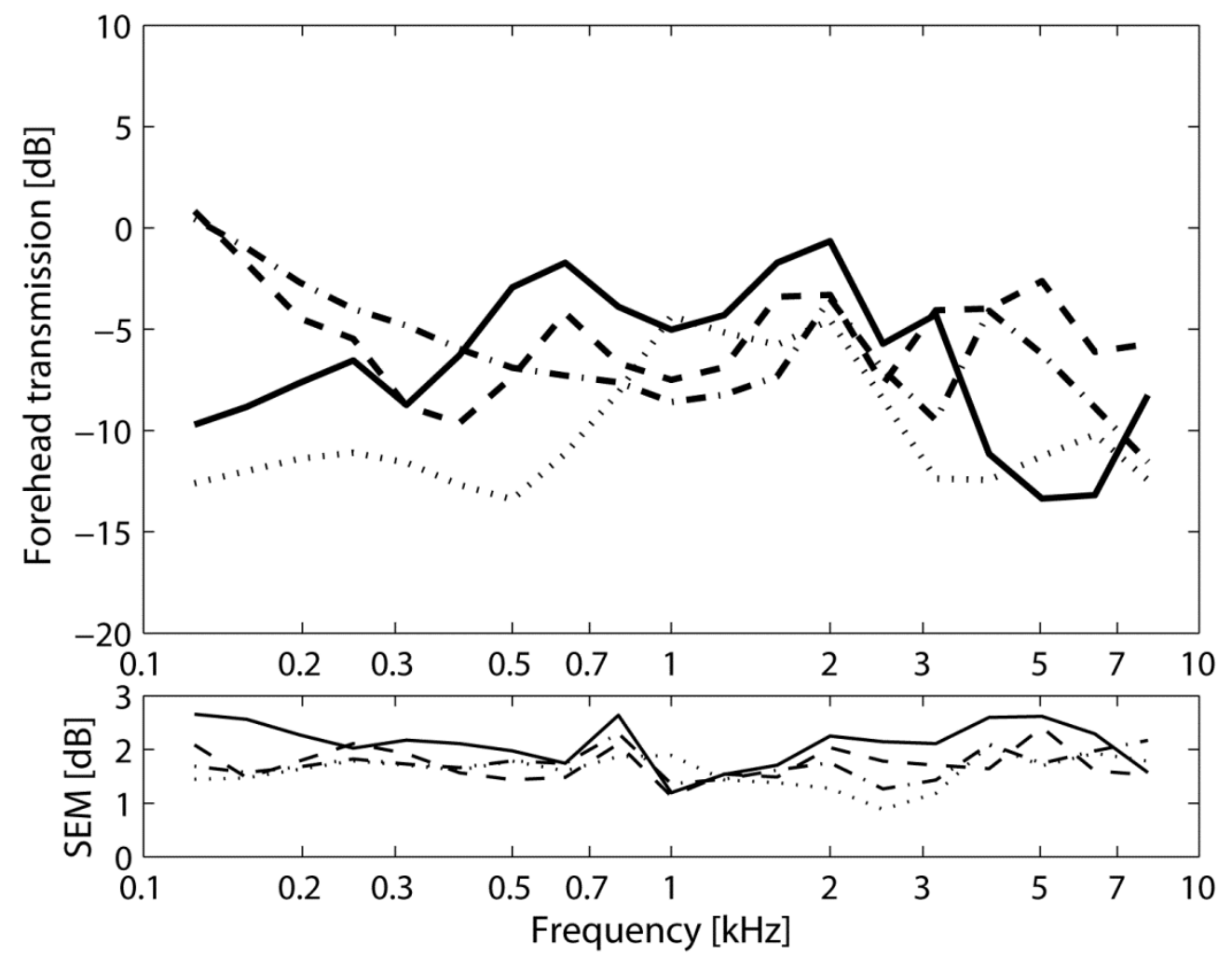

Similar to the transcranial transmission, the estimate of the forehead transmission depended on the frequency as well as on the method. For all estimation methods and for most frequencies, the forehead transmission fell between -10 and $-5 \mathrm{~dB}$, but for some frequencies it extended to be within -13 and $1 \mathrm{~dB}$. Compared to the transcranial transmission, the SDs for the forehead transmission were slightly larger. The SD for the ECSP with open ear-canal was the overall greatest whereas the hearing thresholds with open ear-canal showed the generally lowest SD. However, the difference in SD between the four estimation methods was low and the SD was between 4 and $12 \mathrm{~dB}$ for all frequencies. 
In the same way as the transcranial transmission, the results of hearing thresholds and ECSP from the open and occluded ear-canals differed at low frequencies, while they were more similar at higher frequencies, above $0.8 \mathrm{kHz}$. When investigating frequencies and conditions that produced a statistically significant different forehead transmission (Table 1), a similar result as with the transcranial transmission was found (also Table 1). When estimated by hearing thresholds, the open condition produced statistically significantly lower forehead transmission estimates for frequencies at and below $0.5 \mathrm{kHz}$ (6 to $13 \mathrm{~dB}, \mathrm{p}<0.01$ ) than the occluded condition. There also was statistically significantly lower transmission as estimated by the open ear thresholds at $4 \mathrm{kHz}$ $(\mathrm{p}<0.01)$ as compared with the occluded condition. With the same comparison made for ECSP,

the open ear-canal gave lower forehead transmission at the lowest frequency $(0.125 \mathrm{kHz}, \mathrm{p}<0.01)$ but was greater by about $3 \mathrm{~dB}$ at $0.5 \mathrm{kHz}(\mathrm{p}<0.01$, Table 1$)$. At the higher frequencies, at 4 and 6 $\mathrm{kHz}$, the open ear-canal produced forehead transmission estimates that were statistically significantly lower than for the occluded ear-canal. When the estimates were compared between thresholds and ECSP, almost identical statistical significances were found for the open ear condition with differences at $0.5-0.75 \mathrm{kHz}$ and $2-3 \mathrm{kHz}$ while the occluded condition only gave weak differences at 3 and $8 \mathrm{kHz}(\mathrm{p}<0.05)$. Consequently, for both transcranial transmission and forehead transmission, the method (hearing thresholds or ECSP) produced similar transmission estimates while the condition (open or occluded) affected the transmission estimate; primarily at the low but also to some extent at higher frequencies.

\subsection{Occlusion effect}

The occlusion effect is shown in Fig. 5a for the ECSP measurements and in Fig. 5b for the BC hearing thresholds in the frequency range 0.125 to $4 \mathrm{kHz}$. The results from the contralateral 
measurements are shown with solid lines, the results from the ipsilateral measurements with dashed lines, and the results from the forehead stimulations with dotted lines. The occlusion effect with contralateral and forehead stimulation measured by the ECSP (Fig. 5a) had a maximum of 25 to $30 \mathrm{~dB}$ at the lowest frequencies $(0.125$ to $0.3 \mathrm{kHz})$ that decreased as a function of frequency and became approximately $-10 \mathrm{~dB}$ at $3 \mathrm{kHz}$. For occlusion effect with contralateral and forehead stimulation measured as threshold shifts, the maximum values were slightly more than $20 \mathrm{~dB}$ at 0.2 to $0.3 \mathrm{kHz}$ and decreased as frequency increased to finally stay close to $0 \mathrm{~dB}$ at $2 \mathrm{kHz}$ and above. When the stimulation was at the ipsilateral mastoid, the threshold occlusion effect was 5 to $10 \mathrm{~dB}$ lower at frequencies below $0.8 \mathrm{kHz}$ than when the stimulation was at the other two positions. This difference was statistically significant at and below $0.5 \mathrm{kHz}(\mathrm{p}<0.01$, Table 2). At frequencies from $1.0 \mathrm{kHz}$ and above, the threshold occlusion effects were close with no influence of stimulation position. A low-frequency difference of almost $10 \mathrm{~dB}$ was also seen for the ECSP occlusion effect where the ipsilateral stimulated occlusion effect was statistically significantly $(\mathrm{p}<0.01)$ lower than the other two. However, this appeared only at the lowest frequency measured $(0.125 \mathrm{kHz})$. Moreover, the forehead stimulated occlusion effect at $0.5 \mathrm{kHz}$ was statistically significantly lower than the other two $(\mathrm{p}<0.01$, Table 2$)$; this difference was only a couple of $\mathrm{dB}$ and was not considered important. 
Figure $5 a$
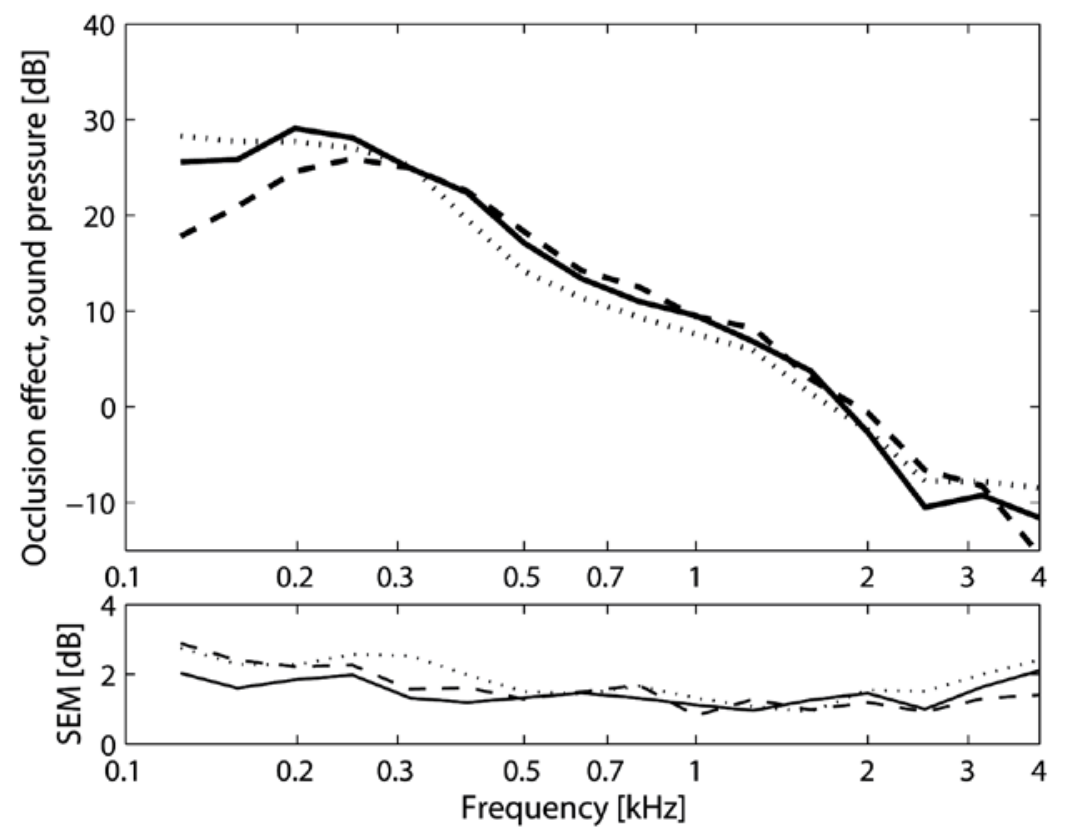

Figure $5 b$

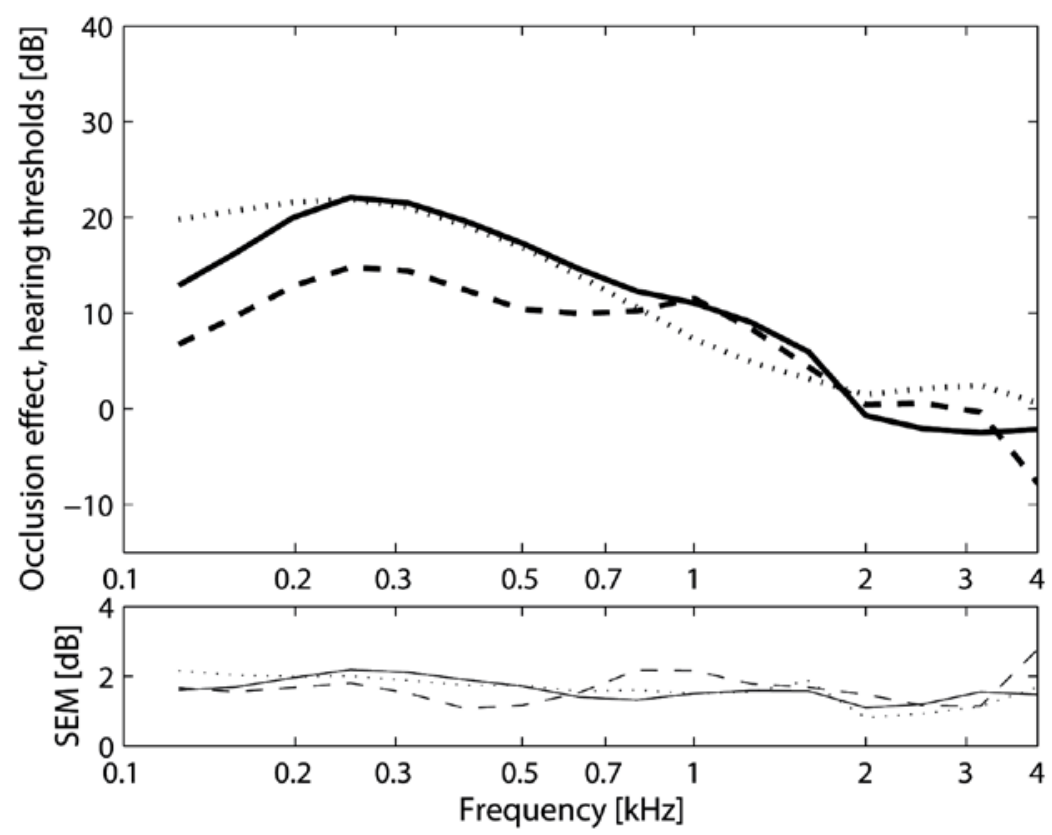


Table 2: Statistical significance according to two-tailed paired t-test for BC occlusion effect measurement. $I=$ ipsilateral stimulation; $C=$ contralateral stimulation; $F=$ forehead stimulation; $N S$ = non-significant

\begin{tabular}{|c|c|c|c|c|c|c|c|c|c|}
\hline \multirow[b]{3}{*}{ Frequency [Hz] } & \multicolumn{6}{|c|}{ Stimulation position } & \multicolumn{3}{|c|}{ Hearing thresholds vs ECSP } \\
\hline & \multicolumn{3}{|c|}{ Hearing thresholds } & \multicolumn{3}{|c|}{ ECSP } & & & \\
\hline & I vs $\mathrm{C}$ & I vs F & C vs F & I vs $C$ & I vs F & $\mathrm{C}$ vs $\mathrm{F}$ & I & $\mathrm{C}$ & $\mathrm{F}$ \\
\hline 125 & $\mathrm{p}<0.01$ & $\mathrm{p}<0.01$ & $\mathrm{p}<0.01$ & $\mathrm{p}<0.01$ & $\mathrm{p}<0.01$ & NS & $\mathrm{p}<0.01$ & $\mathrm{p}<0.01$ & $\mathrm{p}<0.05$ \\
\hline 250 & $\mathrm{p}<0.01$ & $\mathrm{p}<0.01$ & NS & NS & NS & NS & $\mathrm{p}<0.01$ & $\mathrm{p}<0.05$ & NS \\
\hline 500 & $\mathrm{p}<0.01$ & $\mathrm{p}<0.01$ & NS & NS & $\mathrm{p}<0.01$ & $\mathrm{p}<0.01$ & $\mathrm{p}<0.01$ & NS & NS \\
\hline 750 & NS & NS & NS & NS & NS & NS & NS & NS & NS \\
\hline 1000 & NS & NS & NS & NS & NS & NS & NS & NS & NS \\
\hline 1500 & NS & NS & $\mathrm{p}<0.05$ & NS & NS & $\mathrm{p}<0.05$ & NS & NS & NS \\
\hline 2000 & NS & NS & NS & NS & NS & NS & NS & NS & $\mathrm{p}<0.05$ \\
\hline 3000 & NS & NS & $\mathrm{p}<0.05$ & NS & NS & NS & $\mathrm{p}<0.01$ & $\mathrm{p}<0.01$ & $\mathrm{p}<0.01$ \\
\hline 4000 & NS & $\mathrm{p}<0.01$ & NS & NS & $\mathrm{p}<0.05$ & NS & $\mathrm{p}<0.05$ & $\mathrm{p}<0.01$ & $\mathrm{p}<0.01$ \\
\hline
\end{tabular}

At the lower frequencies, the occlusion effects were higher from the ECSP measurements than from the threshold measurements (cf Figs. 5a and 5b). This observation was supported by the ttests displayed in Table 2 where ipsilateral stimulation showed statistically significant differences at $0.5 \mathrm{kHz}$ and below, contralateral stimulation showed statistically significant differences at and below $0.25 \mathrm{kHz}$ while forehead stimulation only produced weak statistically significant difference at the lowest frequency $(0.125 \mathrm{kHz})$. There were also differences at the higher frequencies where the ECSP data were around $-10 \mathrm{~dB}$ while the threshold data were close to 0 $\mathrm{dB}$; this was also supported by the t-test shown in Table 2.

The SD of the occlusion effect estimations were generally between 5 and $10 \mathrm{~dB}$ for all. For the ECSP, there were slightly larger SDs at the frequencies below $0.3 \mathrm{kHz}$ while they were close to 5 $\mathrm{dB}$ between 1 and $3 \mathrm{kHz}$. For the threshold estimates, the SDs were close to 7-8 dB for almost the whole frequency range. 


\section{Discussion}

The hypothesis was that due to the proximity of the bone surrounding the ear-canal and the bone encapsulating the cochlea, the ECSP might be used to estimate the cochlear perception during BC stimulation in normal hearing subjects. This hypothesis was investigated by measurements of hearing thresholds and ECSP with BC stimulation at three different positions, widely spread over the head. The means of these measurements were presented in Figs. 3 and 4 and they displayed both similarities and differences. Consequently, the ECSP could not be used for estimating changes in perception of BC sound for the entire frequency range tested ( $0.125 \mathrm{to} 8 \mathrm{kHz})$, but it could be used for most frequencies $(0.125-0.25,1-1.5$, and $4-8 \mathrm{kHz})$.

It should be emphasized that only normal hearing subjects were tested in this study. For subjects with lesions of the outer and middle ear, the results may be different. Also, the occlusion effect depends on the insertion depth of the ear-plug and the results here are based on an insertion depth of $18 \mathrm{~mm}$ from tragus for all subjects.

\subsection{Difference between ear-canal sound pressure and hearing thresholds}

To investigate the similarities of the measurements further, the individual differences between ECSP and threshold estimates of skull vibration transmission were analyzed and presented in Table 3. The differences are presented as means and standard deviations from the 20 participants. Mean results close to $0 \mathrm{~dB}$ suggest that the group data indicate similar vibration response at the ear-canal and the cochlea while a low SD (close to $0 \mathrm{~dB}$ ) indicates that the mean results hold for individual subjects. 
Table 3: Mean difference and standard deviation (SD) between ECSP and hearing thresholds. The data are presented as [mean (SD)] in $d B$.

\begin{tabular}{|c|c|c|c|c|c|c|c|}
\hline \multirow[b]{2}{*}{ Frequency $[\mathrm{Hz}]$} & \multicolumn{2}{|c|}{ Transcranial transmission } & \multicolumn{2}{|c|}{ Forehead transmission } & \multicolumn{3}{|c|}{ Occlusion effect } \\
\hline & Open & Occluded & Open & Occluded & Ipsi & Contra & Forehead \\
\hline 125 & $0.0(8.6)$ & $0.9(6.7)$ & $2.9(15.2)$ & $0.4(8.0)$ & $11.1(12.5)$ & $12.7(10.5)$ & 8.5 (15.9) \\
\hline 250 & $3.4(10.6)$ & $-2.2(6.5)$ & $4.6(12.2)$ & $-1.5(4.2)$ & $11.1(10.4)$ & $6.0(12.5)$ & $5.1(14.2)$ \\
\hline 500 & $9.7(8.9)$ & $1.1(5.3)$ & $10.5(10.0)$ & $-0.5(8.7)$ & $7.9(7.8)$ & $-0.2(10.2)$ & $-2.8(9.7)$ \\
\hline 750 & $3.7(9.4)$ & $0.4(5.6)$ & $4.2(11.9)$ & $0.9(7.3)$ & $2.3(10.4)$ & $-1.3(7.7)$ & $-1.2(7.9)$ \\
\hline 1000 & $0.3(7.6)$ & $0.8(3.7)$ & $-0.7(10.9)$ & $1.1(4.5)$ & $-2.1(8.7)$ & $-1.5(7.2)$ & $0.3(9.5)$ \\
\hline 1500 & $2.2(5.6)$ & $0.4(6.8)$ & $4.0(7.6)$ & $3.9(7.8)$ & $-1.5(9.6)$ & $-2.2(10.6)$ & $-1.8(10.2)$ \\
\hline 2000 & $5.3(7.3)$ & $3.6(8.8)$ & $3.9(8.3)$ & $0.2(5.7)$ & $-1.1(8.9)$ & $-2.0(8.7)$ & $-3.9(8.2)$ \\
\hline 3000 & $6.4(8.4)$ & $6.2(10.0)$ & $8.1(10.8)$ & $5.4(10.0)$ & $-7.9(7.4)$ & $-6.8(8.9)$ & $-10.3(8.6)$ \\
\hline 4000 & $2.9(10.0)$ & $-1.4(11.4)$ & $1.3(14.6)$ & $0.1(12.2)$ & $-7.7(13.3)$ & $-9.4(10.0)$ & $-9.1(13.3)$ \\
\hline 6000 & $-2.8(11.8)$ & $5.6(10.1)$ & $-2.9(14.1)$ & $2.7(12.8)$ & $-11.0(9.9)$ & $-0.1(13.7)$ & $-5.2(14.2)$ \\
\hline 8000 & $6.7(6.9)$ & $9.7(7.8)$ & 4.2 (11.6) & $5.8(10.6)$ & $-2.3(10.8)$ & 0.9 (10.7) & $2.7(13.7)$ \\
\hline
\end{tabular}

Most of the mean differences in Table 3 that were greater than $3 \mathrm{~dB}$ were statistically significant while none that were less than $3 \mathrm{~dB}$ were statistically significant. Therefore, if a mean difference of $3 \mathrm{~dB}$ was used as a limit, slightly less than half of the tested frequencies with open ear-canals met the criterion (below the $3 \mathrm{~dB}$ limit), both for transcranial (5 of 11 tested frequencies) and forehead transmission (4 of 11). When the ear-canal was occluded, a majority of the tested frequencies had a mean difference of less than $3 \mathrm{~dB}$ (7 of 11 for transcranial and 8 of 11 for forehead transmission). This suggested that there was a general agreement between ECSP and hearing thresholds when the ear-canal was occluded but not when it was open. However, it 
should be noted that there were only a few mean difference results that were more than $6 \mathrm{~dB}$ regardless of open or occluded ear-canal. When investigating the difference results in Table 3 more closely, it was clear that the skull transfer estimations by ECSP were, for all conditions and most frequencies, greater than that obtained with hearing thresholds (i.e. positive values in Table 3). No difference had negative values that exceeded $3 \mathrm{~dB}$.

A difference of more than $3 \mathrm{~dB}$ for the open ear-canal transmissions was found at 0.25 to 0.75 $\mathrm{kHz}$ (Table 3) where the differences at 0.5 and $0.75 \mathrm{kHz}$ were statistically significant according to Table 1. Similar differences were not found when the ear-canal was occluded. One possible explanation for this is that the hearing thresholds with ipsilateral stimulation were improved compared to the other stimulation positions. According to Fig. 3 the difference between ECSP and thresholds originated in the threshold measurements where a dip in the transcranial transmission was seen at $0.5 \mathrm{kHz}$ whereas the ECSP were similar irrespective if stimulation was ipsilateral or contralateral. The relatively higher sensitivity from ipsilateral stimulation was not easily explainable when similar results were neither seen in the current ECSP measurement nor in cochlear vibration measurement executed previously in cadaver heads (Stenfelt and Goode, 2005a). However, in the Stenfelt and Goode (2005a) study it was concluded that the response vibration direction of the cochlea was different when the stimulation was close (ipsilateral mastoid) than when it was further away (e.g. forehead or contralateral mastoid). Consequently, the explanation may be that the cochlea was more sensitive to the vibration direction caused by the ipsilateral stimulation compared to other directions. According to Kim et al. (2011), based on simulations of a finite element model of the middle ear and cochlea, the basilar membrane vibrates for all BC stimulation directions. However, they made their analysis in a simplified 
geometry and with pure rigid body motion and the results cannot be generalized for analyzing the directional sensitivity of the human cochlea during BC stimulation.

There were statistically significantly greater transmission estimates for ECSP than thresholds for all conditions at $3 \mathrm{kHz}$ (Tables 1 and 3). At this frequency, the occlusion effect did not influence the perceptual response (Fig. 5b). Since a similar effect was seen for both transcranial transmission and forehead transmission, the origin was probably in the ipsilateral responses. One possible explanation was, once again, based on the $\mathrm{BC}$ response direction from ipsilateral stimulation, but at this frequency it was caused by the vibration of the middle ear ossicles. It has been shown that the ossicles' vibration is higher when the stimulation is in line with the lowfrequency vibration direction of the ossicles than for the perpendicular directions (Stenfelt et al., 2002). Also, it has been suggested that the ossicles can contribute to the perceived BC sound in the 2 to $3 \mathrm{kHz}$ frequency range (Stenfelt and Goode, 2005b; Stenfelt, 2006). Consequently, the difference seen at $3 \mathrm{kHz}$, and to some extent at $2 \mathrm{kHz}$, may be due to the contribution of the ossicles when the stimulation was at the ipsilateral mastoid compared to less or nonexistent ossicle contribution when the stimulation was at the other positions. This hypothesis has some support from clinical findings where approximately $5 \mathrm{~dB}$ worse $\mathrm{BC}$ hearing thresholds were reported at frequencies between 0.5 to $4 \mathrm{kHz}$ when the $\mathrm{BC}$ transducer was positioned at the mastoid compared with the forehead in patients with middle ear pathologies (Dirks et al., 1969; Goodhill et al., 1970; Studebaker, 1962). The greater frequency range (0.5 to $4 \mathrm{kHz}$ ) for the sensitivity difference reported in the above studies was not found here. This difference may originate in the middle ear pathology itself. As the BC perception is the sum of different pathways, the pathways applicable with a middle ear pathology (the above studies) may be different from the pathways in a normal ear (current study). 
There was a 5 to $10 \mathrm{~dB}$ difference between ECSP and threshold based transcranial transmission at 6 and $8 \mathrm{kHz}$ when the ear-canal was occluded (Table 3); the effect was not that large in the forehead transmission. There was a slightly lower difference in the transcranial transmission (7 $\mathrm{dB})$ at $8 \mathrm{kHz}$ when the ear-canal was open. It was therefore likely that the difference originated from the contralateral stimulation (much smaller difference in the forehead transmission). However, at present, we cannot present a good explanation for the phenomenon but it is plausible that the contralateral stimulation elicits different pathways for the BC perception than at the forehead and ipsilateral mastoid.

\subsection{Difference between open and occluded results}

The BC transmission difference for the open ear-canal at frequencies between 0.25 and $0.75 \mathrm{kHz}$ discussed above was not seen when the ear-canal was occluded (Table 3); the occluded ECSP based BC transmission estimates was similar to the occluded threshold based estimations. This similarity originates in the effect of occlusion. When occluded, the ECSP during BC stimulation was increased at frequencies below $2 \mathrm{kHz}$ and dominated the perceptual BC response (Stenfelt et al., 2003a; Stenfelt and Reinfeldt, 2007). Consequently, the similarity at these frequencies was not a result of the ECSP reflecting the BC perceptual response but the opposite; the BC perceptual response was caused by the ECSP. This means that the occluded ECSP was not a true estimate of the perceptual BC response at low frequencies. However, if the occlusion is deep enough to avoid an occlusion effect, the occluded data may resemble the open ear-canal data.

When inspecting Figs. 3 and 4 together with Table 1 it was found that the condition of open and occluded ear-canal affected the estimation of BC sound transmission in the skull. When hearing thresholds were used the statistically significant differences were mainly at frequencies of 0.5 $\mathrm{kHz}$ and below. This was, once again, caused by the occlusion effect. As described in the 
previous paragraph, occluding the ear-canal causes the low-frequency BC sound perception to depend on the ECSP. This is not the case with open ear canal where other pathways than the ECSP determine the perception of BC sound. Accordingly, the estimation of BC sound transmission was different with the ear-canal open and occluded at frequencies of $0.5 \mathrm{kHz}$ and below. When the open and occluded ECSP estimates were compared (Table 1), the most statistically significant differences were at frequencies between 2 and $6 \mathrm{kHz}$. These differences may be caused by airborne sound radiation from the transducer that enter the open but not occluded ear-canal and influences the ECSP when stimulation was at the ipsilateral mastoid (Shipton et al., 1980).

A possible explanation for the low-frequency difference between open and occluded ECSP estimates was the relative importance of the soft tissue and cartilage part of the ear-canal for the ECSP. It has been suggested that this part was more stimulated when the BC transducer was at the ipsilateral mastoid than at other positions on the skull (Stenfelt et al., 2003a). When occluding the ear-canal, part of the sound caused by the soft tissue and cartilage part of the ear-canal was removed due to the ear-plug in that part of the ear-canal. Consequently, the relation between ipsilateral stimulation and other position's influence on the ECSP may change due to the ear-plug insertion depth.

\subsection{Occlusion effects at different stimulation positions}

That there is a difference between perceived occlusion effect and changes of the ECSP has been well documented. It has often been reported that the perceived occlusion effect was about $10 \mathrm{~dB}$ lower than the corresponding change of ECSP (Berger et al., 1983; Huizing, 1960; Reinfeldt et al., 2007; Stenfelt and Reinfeldt, 2007). These comparisons between perceived occlusion effect and ECSP changes relate to frequencies below $2 \mathrm{kHz}$ where the occlusion effect was present. 
Stenfelt et al (2003b) states that when the ear-canal is open, the outer ear contribution to the BC hearing is approximately $10 \mathrm{~dB}$ lower than the other contributions at frequencies below $2 \mathrm{kHz}$, but an occluded ear-canal dominates the low-frequency BC hearing perception. Consequently, the occlusion effect is overestimated when calculated from the ECSP measurements compared to threshold data.

The current data also showed greater ECSP occlusion change than corresponding alteration of threshold data; the greatest difference was found at the lowest frequency $(12.7 \mathrm{~dB}$ at $0.125 \mathrm{kHz}$ for contralateral stimulation, Table 3) that diminished at higher frequencies. The difference between ECSP and threshold occlusion effect also depended on the stimulation position: for ipsilateral stimulation the difference was present at frequencies of $0.5 \mathrm{kHz}$ and below, while the difference was only seen at the two lowest frequencies $(0.125$ and $0.25 \mathrm{kHz})$ when stimulation was at the forehead or contralateral mastoid (Tables 2 and 3). At the higher frequencies, above 2 $\mathrm{kHz}$, there was a general trend of lower ECSP occlusion effect values when compared with the threshold based occlusion effect for all three stimulation positions (Table 3). This was due to the reduction of ECSP caused by the occlusion at higher frequencies, while perception of BC sound was not influenced by occlusion (and therefore insensitive to the decrease in ECSP) due to the contribution of other BC pathways to stimulus perception (Stenfelt and Goode, 2005b; Stenfelt and Reinfeldt, 2007).

When the amount of occlusion effect was analyzed in relation to stimulation position (Fig. 5 and Table 2) the occlusion effect was statistically significantly lower for ipsilateral stimulation than for the other stimulation positions: for the threshold occlusion effect at frequencies of $0.5 \mathrm{kHz}$ and below, while the ECSP occlusion effects only differed at $0.125 \mathrm{kHz}$. The difference seen in the threshold occlusion effect at $0.125 \mathrm{kHz}$ could be explained by differences in ECSP related to 
stimulation position. However, the origin of this difference was not completely clear. It may be speculated that the soft tissues were more excited at the low frequencies when the transducer was close to the ear-canal (ipsilateral mastoid) than when positioned further away, as discussed above. Due to the reduction of soft tissue contribution to the ECSP when the ear-plug was positioned in the soft tissue and cartilage part of the ear-canal, there was a reduction of occluded ECSP for stimulation close to the ear-canal that was not present for other stimulation positions.

The difference in perceived occlusion effect with stimulation at different positions at frequencies above $0.125 \mathrm{kHz}$ cannot be explained by differences in ECSP. This difference was believed to originate in the same difference as discussed above where stimulation close to the cochlea produced a cochlear response direction that was more effective than when the stimulation was further away. Trends of less perceived occlusion effect when the stimulation is at the mastoid compared with other positions have been indicated previously (Dean et al., 2000; Klodd et al., 1977; Stenfelt and Reinfeldt, 2007). Stenfelt and Goode (2005a) divided the BC transmission of the head into three frequency ranges: (1) below approximately $0.3 \mathrm{kHz}$ the skull moves as a true rigid body, (2) between 0.3 and $1 \mathrm{kHz}$ the skull motion can be approximated by a mass-spring system, and (3) above $1 \mathrm{kHz}$ the skull moves in all directions determined by the wave transmission in the skull bone. Accordingly, the vibration response of the skull bone is more or less in the stimulation direction for frequencies below $1 \mathrm{kHz}$ and, consequently, the vibrations of the outer, middle, and inner ear are different for forehead and ipsilateral stimulation. Therefore, the lower perceived occlusion effect from ipsilateral stimulation may be explained by the different BC contributing parts' relative efficiency depending on the stimulation positions. 


\subsection{Comparisons with previous measurements}

Transcranial transmission estimates, or its reciprocal, transcranial attenuation, have earlier been reported based on hearing thresholds or vibrations measurements. Here, we add another method: measurement of ECSP. The data were obtained for two conditions, with the ear-canal open and occluded. As discussed previously, using occluded ear-canals does not necessarily reflect normal $\mathrm{BC}$ perception due to the occlusion effect and the alteration of dominant $\mathrm{BC}$ pathways.

The results in this study were compared with similar measurements from some previous studies. The transcranial transmission estimates by ECSP with both open (solid line) and occluded earcanals (dashed line) were compared with cochlear vibration measurements in Fig. 6a. The cochlear vibration data were obtained as the vibration of the cochlear promontory using a laser Doppler vibrometer in one dimension (dotted line, Eeg-Olofsson et al. (2011)) and the energy summation of three dimension using accelerometers (dashed-dotted line, Stenfelt and Goode (2005a)) in human cadaver heads. In the cochlear vibration measurements the transducer was rigidly attached to the mastoid bone at a similar position as in the current study but without any skin and subcutaneous tissues between the transducer and skull bone. According to Fig. 6a, the vibration data and ECSP data were generally within $5 \mathrm{~dB}$ independent of condition and type of measurement. The greatest deviation was found at 0.5 to $0.7 \mathrm{kHz}$ where the transcranial transmission estimate by one-dimensional cochlear vibration was greater than the others. Otherwise, the general trend was that the transcranial transmission was close to $0 \mathrm{~dB}$ at frequencies below $1 \mathrm{kHz}$, decreased at higher frequencies and became around $-10 \mathrm{~dB}$ at the highest frequencies. The similarities between the cochlear vibration and ECSP data suggested that the vibration of the cochlea and ear-canal was similar. 
Figure 6a

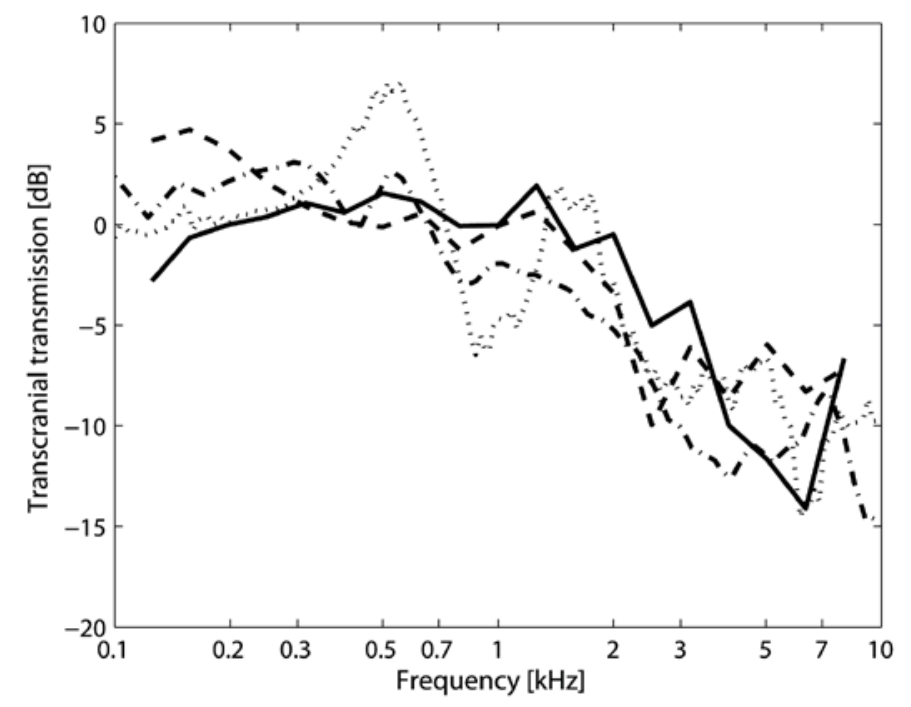

Figure $6 b$

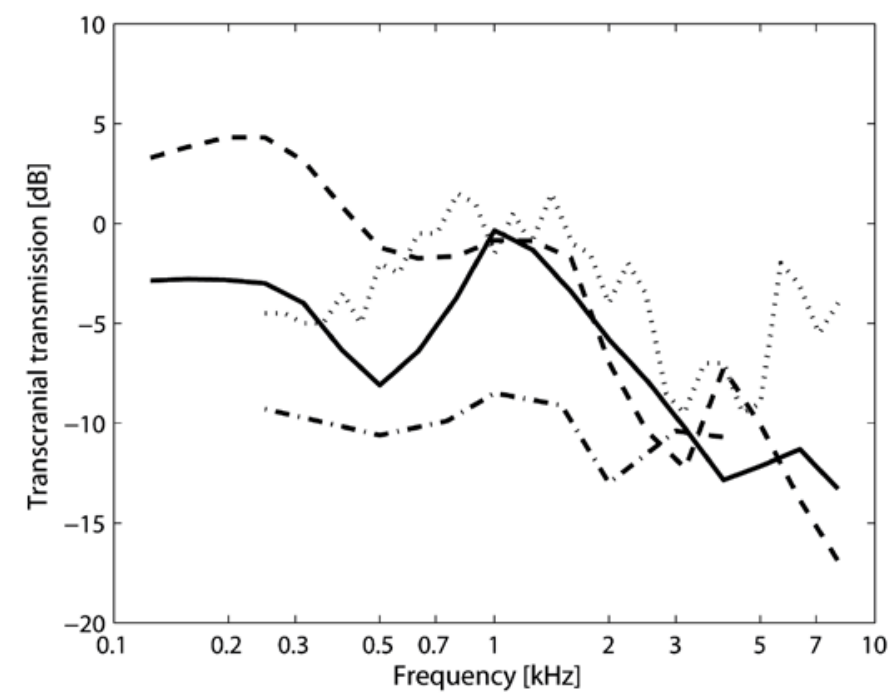


Figure 6c

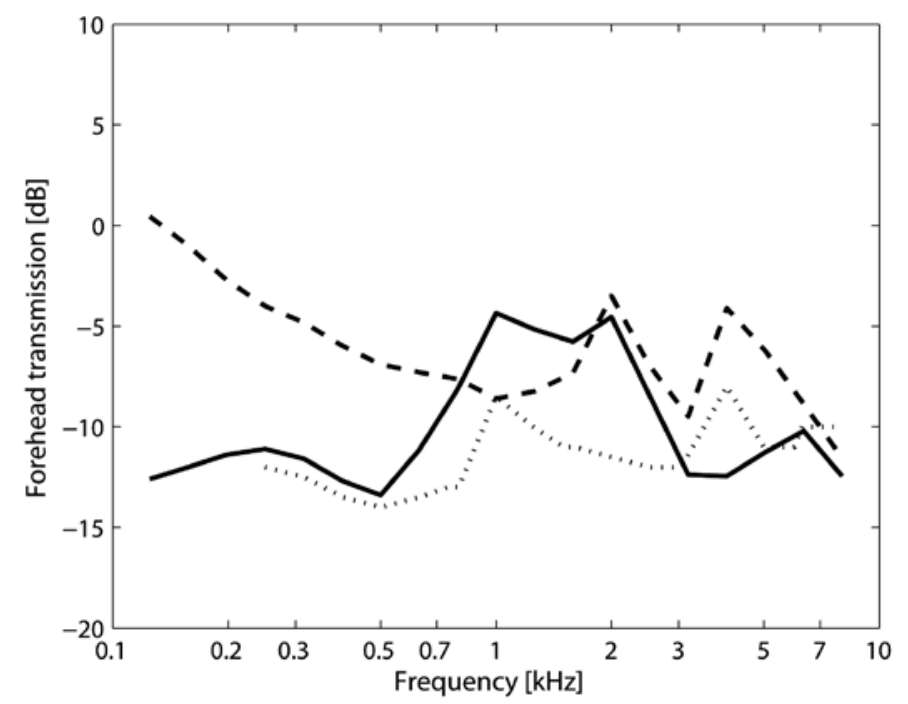

The transcranial transmission estimates using threshold data with open (Fig. 6b, solid line) and occluded ear-canals (Fig. 6b, dashed line) were compared with other published transcranial transmission estimates. In Fig. 6b the recently published results from 28 unilateral subjects (dotted line, Stenfelt (2012)) were included as well as the data from both normal hearing subjects and unilateral deaf subjects by Nolan and Lyon (1981) (dash-dotted line). As seen previously, there was a substantial difference at low frequencies with open and occluded ear-canals; at frequencies of $1 \mathrm{kHz}$ and above the two ways of estimating the transcranial transmission was similar. It can also be observed that the estimate from Nolan and Lyon (1981) was distinctively lower than from the other studies; it was only at 3 and $4 \mathrm{kHz}$ that it was similar to the other three estimates. Even if they were not completely equal, the open ear estimate in this study was similar to the also open ear estimate in Stenfelt (2012). There was a clear difference at frequencies of 6 $\mathrm{kHz}$ and above where the Stenfelt (2012) estimate showed nearly $10 \mathrm{~dB}$ less separation between the sides than the estimates in the current study. There were differences in the measurement setup 
between the two studies, e.g. in type of BC transducer and requirement of masking. However, it was not obvious that any of those differences influenced the data at the highest frequencies.

A third comparison was shown in Fig. 6c where the forehead transmission estimate using threshold data (open ear, solid line; occluded ear, dashed line) was shown together with the interim differences in reference equivalent threshold force levels (RETFL) between forehead and mastoid location of a vibrator (dotted line, ISO:389-3 (1994)). Most of the RETFL difference data in the standard are obtained from three studies, but some of the values are only obtained from one study. Once again, there was a clear difference between occluded and open ears at frequencies below $1 \mathrm{kHz}$, where the open ear data were close to the forehead sensitivity stated in the standard (ISO:389-3, 1994). And, except at frequencies between 1 and $2 \mathrm{kHz}$, there was a good correspondence between the forehead sensitivity given in the standard and the open ear data in this study.

The occlusion effect has been estimated for various types of occlusion devices numerous times. The exact amount of occlusion depends on the type of occlusion device and its exact location (Stenfelt and Reinfeldt, 2007). It was therefore not meaningful to compare the exact occlusion effect in this study with others. However, the occlusion effects displayed in Fig. 5 were similar to others that have measured the occlusion effect as both alteration of the ECSP and as alteration of hearing thresholds (Berger et al., 1983; Huizing, 1960; Stenfelt and Reinfeldt, 2007). 


\section{Conclusions}

Hearing thresholds and ECSP were used to estimate BC sound transmission in the human skull. When the ear-canal was open, the BC skull transmission estimates from hearing thresholds and ECSP were similar at most of the tested frequencies. The exception was for the frequencies 0.5 , $0.75,2$ and $3 \mathrm{kHz}$, where statistically significant differences were found. This means that even if ECSP could estimate the BC sound transmission to the cochlea for most frequencies, such method could cause errors of up to $10 \mathrm{~dB}$ at a few frequencies.

When comparing open and occluded conditions for BC skull transmission estimates, statistically significant differences were found mainly at the low frequencies. These differences were caused by ECSP becoming the dominant contributor for BC perception with occluded ear-canal which is not the case when the ear-canal is open. Hence, occluded measurements should be avoided for estimation of normal BC perception, at least for occlusion devices and depths used in this study.

The ECSP gave similar result as previously reported cochlear promontory vibration measurements for BC skull transmission, supporting the hypothesis of similar relative vibration level at the cochlea and ear-canal (cf Fig. 6a).

Furthermore, the results from both BC skull transmissions and occlusion effects showed that the sensitivity of the different contributors to BC perception depended on stimulation position and most probably on the vibration direction. Specifically, a greater sensitivity of the cochlea for vibration in line with the ipsilateral stimulation direction, compared to contralateral and forehead stimulation, was indicated. 


\section{Acknowledgements}

This study was supported by grant from the Swedish Research Council (621-2002-5624). 


\section{Captions}

Figure 1: Measurement setup for the ECSP measurements: The BC transducer was fed via an amplifier A from a Brüel \& Kjær Pulse signal analyzer. Input 1 of the signal analyzer was connected to the output from the amplifier while input 2 was connected to the probe tube microphone. The test subject together with the probe tube microphone and the BC transducer were situated in a sound attenuated room.

Figure 2: (a) The transcranial transmission was calculated as the difference in ECSP levels or BC hearing thresholds between contralateral and ipsilateral mastoid stimulation. (b) The forehead transmission was calculated as the difference in ECSP levels or the BC hearing thresholds between stimulation at the forehead and the ipsilateral mastoid.

Figure 3: The transcranial transmission mean estimates obtained by ECSP measurements with open (solid line) and occluded ear-canals (dashed line), and obtained by BC hearing thresholds with open (dotted line) and occluded ear-canals (dash-dotted line). The SD result uses the same line properties.

Figure 4: The forehead transmission mean estimates obtained by ECSP measurements with open (solid line) and occluded ear-canals (dashed line), and obtained by BC hearing thresholds with open (dotted line) and occluded ear-canals (dash-dotted line). The SD result uses the same line properties. 
Figure 5: (a) The mean occlusion effects from the ECSP measurements with contralateral (solid line), ipsilateral (dashed line), and forehead stimulation (dotted line). (b) The occlusion effects from the BC hearing thresholds with contralateral (solid line), ipsilateral (dashed line), and forehead stimulation (dotted line). The SD result uses the same line properties.

Figure 6: (a) The transcranial estimates from the current study using ECSP with open (solid line) and occluded (dashed line) ear-canals are displayed together with transcranial estimates based on cochlear vibration in one dimension (dotted line, Eeg-Olofsson et al. (2011)) and the energy summation in three dimensions (dashed-dotted line, Stenfelt and Goode (2005a)). (b) The transcranial estimates from the current study using hearing thresholds with open (solid line) and occluded (dashed line) ear-canals are displayed together with hearing threshold based transcranial estimates from Stenfelt (2012) (dotted line) and Nolan and Lyon (1981) (dashed-dotted line). (c) Forehead transmission estimates from the current study using hearing thresholds with open (solid line) and occluded (dashed line) ear-canals are displayed together with the same estimate given in the standard ISO:389-3 (1994) (dotted line). 


\section{References}

Bárány, E. 1938. A contribution to the physiology of bone conduction. Acta Oto-laryngologica Suppl 26, 1-223.

Berger, E.H., Kerivan, J.E. 1983. Influence of physiological noise and the occlusion effect on the measurement of real-ear attenuation threshold. J. Acoust. Soc. Am. 74, 81-94.

Dean, M.S., Martin, F.N. 2000. Insert earphone depth and the occlusion effect. American Journal of Audiology 9, 131-134.

Dirks, D., Malmquist, C. 1969. Comparison of frontal and mastoid bone-conduction thresholda in various conductive lesions. Journal of Speech and Hearing Research 12, 725-746.

Eeg-Olofsson, M., Stenfelt, S., Tjellström, A., Granström, G. 2008. Transmission of boneconducted sound in the human skull measured by cochlear vibrations. International Journal of Audiology 47, 761-769.

Eeg-Olofsson, M., Stenfelt, S., Granström, G. 2011. Implications for contralateral bone conducted transmission as measured by cochlear vibrations. Otology and Neurotology 32, 192-198.Freeman, S., Sichel, J.-Y., Sohmer, H. 2000. Bone conduction experiments in animals - evidence for a non-osseous mechanism. Hearing Research 146, 72-80.

Goodhill, V., Dirks, D., Malmquist, C. 1970. Bone-conduction thresholds. Relationships of frontal and mastoid measurement in conductive hypacusis. Archives Otolaryngology 91, 250-256.

Homma, K., Du, Y., Shimizu, Y., Puria, S. 2009. Ossicular resonance modes of the human middle ear for bone and air conduction. J. Acoust. Soc. Am. 125, 968-979.

Huizing, E. 1960. Bone conduction - The influence of the middle ear. Acta Oto-Laryngolica. Suppl 155, 1-99.

Håkansson, B. 2003. The balanced electromagnetic separation transducer: A new bone conduction transducer. J. Acoust. Soc. Am. 113, 818-825.

Håkansson, B., Reinfeldt, S., Eeg-Olofsson, M., Ostli, P., Taghavi, H., Adler, J., Gabrielsson, J., Stenfelt, S., Granström, G. 2010. A novel bone conduction implant (BCI): engineering aspects and pre-clinical studies. International Journal of Audiology 49, 203-215.

ISO:226. 2003. International Organization for Standardization. Acoustics - Normal equal loudness-level contours. Geneva.

ISO 389-1. 1998. International Organization for Standardization. Acoustics - Reference zero for the calibration of audiometric equipment - Part 1: Reference equivalent threshold sound pressure levels for pure tones and supra-aural earphones. Geneva. 
ISO:389-3. 1994. International Organization for Standardization. Reference zero for the calibration of audiometric equipment. Part 3: Reference equivalent threshold force levels for pure tones and bone vibrators. Geneva.

ISO:8253-1. 2010. International Organization for Standardization. Acoustics -- Audiometric test methods -- Part 1: Pure-tone air and bone conduction audiometry. Geneva.

Ito, T., Röösli, C., Kim, C., Sim, J., Huber, A., Probst, R. 2011. Bone conduction thresholds and skull vibration measured on the teeth during stimulation at different sites on the human head. Audiology Neurotology 16, 12-22.

Kim, N., Homma, K., Puria, S. 2011. Inertial bone conduction: Symmetric and anti-symmetric components. Journal of the Association for Research in Otolaryngology 12, 261-279.

Klodd, D., Egerton. 1977. Occlusion effect: bone conduction speech audiometry using forehead and mastoid placement. Audiology 16, 522-529.

Nolan, M., Lyon, D.J. 1981. Transcranial Attenuation in Bone Conduction Audiometry. The Journal of Laryngology and Otology 95, 597-608.

Reinfeldt, S., Stenfelt, S., Good, T., Håkansson, B. 2007. Examination of bone-conducted transmission from sound field excitation measured by thresholds, ear-canal sound pressure, and skull vibrations. J. Acoust. Soc. Am. 121, 1576-1587.

Shipton, M.S., John, A.J., Robinson, D.W. 1980. Air-radiated sound from bone vibration transducers and its implications for bone conduction audiometry. British Journal of Audiology 14, 86-99.

Stenfelt, S., Håkansson, B., Tjellström, A. 2000. Vibration characteristics of bone conducted sound in vitro. J. Acoust. Soc. Am. 107, 422-431.

Stenfelt, S., Hato, N., Goode, R. 2002. Factors contributing to bone conduction: The middle ear. J. Acoust. Soc. Am. 111, 947-959.

Stenfelt, S., Wild, T., Hato, N., Goode, R.L. 2003a. Factors contributing to bone conduction: The outer ear. J. Acoust. Soc. Am. 113, 902-912.

Stenfelt, S., Puria, S., Hato, N., Goode, R.L. 2003b. Basilar membrane and osseous spiral lamina motion in human cadavers with air and bone conduction stimuli. Hearing Research 181, 131-143.

Stenfelt, S., Goode, R.L. 2005a. Transmission properties of bone conducted sound: Measurements in cadaver heads. J. Acoust. Soc. Am. 118, 2373-2391.

Stenfelt, S., Goode, R. 2005b. Bone conducted sound: Physiological and clinical aspects. Otology and Neurotology 26, 1245-1261. 
Stenfelt, S. 2006. Middle ear ossicles motion at hearing thresholds with air conduction and bone conduction stimulation. J. Acoust. Soc. Am. 119, 2848-2858.

Stenfelt, S. 2007. Simultaneous cancellation of air and bone conduction tones at two frequencies: extension of the famous experiment by von Békésy. Hearing Research 225, 105-116.

Stenfelt, S., Reinfeldt, S. 2007. A model of the occlusion effect with bone-conducted stimulation. International Journal of Audiology 46, 595-608.

Stenfelt, S. 2011. Acoustic and physiologic aspects of bone conduction hearing. Advances in Oto-Rhino-Laryngology 71, 10-21.

Stenfelt, S. 2012. Transcranial attenuation of bone conducted sound when stimulation is at the mastoid and at the bone conduction hearing aid position. Otology and Neurotology 33, 105-114.

Studebaker, G. 1962. Placement of Vibrator in Bone-Conduction Testing. Journal of Speech and Hearing Research 5, 321-331.

Tonndorf, J. 1966. Bone Conduction. Studies in Experimental Animals. Acta Oto-Laryngologica Suppl, 1-132.

von Békésy, G. 1932. Zur theorie des hörens bei der schallaufnahme durch knochenleitung. Ann. Physik. 13, 111-136.

von Békésy, G. 1948. Vibration of the head in a sound field, and its role in hearing by bone conduction. J. Acoust. Soc. Am. 20, 727-748.

von Békésy, G. 1960. Experiments in hearing. McGraw - Hill, New York. 